\title{
A Minimally Invasive Surgery for Bone Metastases Using the Combination of Photodynamic Therapy and Hyperthermia Treatment
}

\author{
Takao Matsubara ${ }^{1 *}$, Katsuyuki Kusuzaki ${ }^{2}$, Akihiko Matsumine ${ }^{1}$, Kunihiro Asanuma ${ }^{1}$, \\ Tomoki Nakamura ${ }^{1}$, Atsumasa Uchida ${ }^{1}$, Akihiro Sudo ${ }^{1}$ \\ ${ }^{1}$ Department of Orthopaedic Surgery, Mie Graduate School of Medicine, Mie, Japan; ${ }^{2}$ Department of Orthopaedic Surgery, Kujo \\ Hospital, Kyoto, Japan. \\ Email: *takao-m@lin.medic.mie-u.ac.jp
}

Received March 19 ${ }^{\text {th }}, 2013$; revised April 20, th 2013; accepted May 21 ${ }^{\text {st }}, 2013$

Copyright (C) 2013 Takao Matsubara et al. This is an open access article distributed under the Creative Commons Attribution License, which permits unrestricted use, distribution, and reproduction in any medium, provided the original work is properly cited.

\begin{abstract}
Aims: Cancer patients with bone metastases in their extremities may require surgical intervention to prevent deterioration in their quality of life due to a pathological fracture or severe bone pain. However, curative surgical interventions sometimes have severe complications due to the status of the original cancers. To avoid the decreased quality of life caused by bone metastasis, minimally invasive surgery that avoids additional surgical morbidity is required. We have established two therapeutic treatments for bone metastasis, a photodynamic acridine orange treatment (AOT) and an electronic magnetic hyperthermia treatment (EMHT). The present study investigated the clinical outcomes of combination therapy with EMHT and AOT for patients with bone metastases in their extremities. Methods: The study included 6 patients with 7 bone cancer metastasis locations. For bone metastases, all patients received intraregional tumor excision supported by AOT, in which photodynamic and radiodynamic therapy kills tumor cells during surgery with minimal damage to normal tissues. After the curettage, bone reconstruction was performed by using magnetic materials with calcium phosphate cement. EMHT was repeatedly performed after surgery. In EMHT, tumor cells are killed with an electric magnetic field generator, and bony union is promoted by electronic stimulation. Results: The mean duration of follow-up was 14 months. During the follow-up period, only one patient experienced a local recurrence, and this recurrence occurred 14 months after surgery. Bony union occurred in 4 of 5 cases $(80 \%)$, and the pain score was significantly reduced after surgery. Conclusions: In the present study, AOT reduced the invasiveness of surgery. EMHT reduced the tumor growth without major complications and promoted bone formation after surgery. Our clinical results confirmed that EMHT and AOT combination therapy for bone metastasis can preserve limb function without local recurrence or bone absorption.
\end{abstract}

Keywords: Photodynamic Therapy; Minimal Invasive Surgery; Hyperthermia Treatment; Acridine Orange; Bone Metastasis

\section{Introduction}

Patients with bone metastases in their extremities sometimes require surgical intervention to prevent a deterioration in quality of life due to a pathological fracture or severe bone pain [1-6]. Localized radiotherapy with surgical reinforcement has been a standard treatment for bone metastases. However, radiotherapy can cause bone absorption due to tumor regrowth or the lack of bone metabolism $[7,8]$. If a new adjuvant therapy could reduce

"Corresponding author. the surgical invasiveness and surgical margin without an increased incidence of local recurrence, excellent limb function and better quality of life could be maintained after surgery. We have focused on photodynamic therapy (PDT) with photosensitizers as a neoadjuvant therapy. We employed acridine orange (AO) as a photosensitizer to establish AO photodynamic therapy [9-17]. With this technique, tumor cells labeled with $\mathrm{AO}$ are visualized under a fluorescence microscope to allow curettage to be easily performed with minimal damage to normal tissue [18-21]. Labeled tumor cells are killed not only by illu- 
mination but also by a low-dose (5 Gy) radiation beam after surgery [22]. AO therapy eliminates tumor cells without invasiveness and has the potential to reduce this complication by conventional radiation therapy.

Radiation toxicity is another problem associated with conventional radiotherapy for bone metastasis. Complications caused by irradiation, such as fibrosis, skin necrosis, and nerve palsy, can prevent the repeated use of radiotherapy even if a local recurrence occurs.

Our hyperthermia treatment that uses an electronic magnetic generator (EMHT: electronic magnetic hyperthermia treatment using magnetic materials) reduces local recurrence by thermal heating and promotes bone formation after surgery $[23,24]$. EMHT can be repeatedly performed because there are no complications from the thermal heat or electric stimulation.

We established a clinical regimen consisting of photodynamic surgery with AO-labeled tumor cells (AO-PDS); photodynamic therapy with AO (AO-PDT); radiodynamic treatment, in which labeled cells were treated with $\mathrm{X}$-rays (AO-RDT); and EMHT after surgery. The present study examined the clinical outcomes of this regimen in patients with bone metastases.

\section{Patients and Methods}

Between 2003 and 2009, 6 patients with bone metastasis in their extremities were treated with a combination of EMHT and AO treatment (AOT). We selected the patients who required surgical intervention by curettage or marginal resection because AO therapy can be performed during such surgeries. Bone metastases that require curettage are commonly located in the epiphysis. Metastases that extend into extra-osseous lesions can cause massive bone defects. Severe bone defects carry a risk of pathological fracture and sometimes require mass volume reduction by curettage or tumor resection.

\subsection{AO Therapy}

All patients first received intraregional tumor excision with minimal damage to normal tissues, which is similar to conventional tumor excision for benign bone tumors. Photodynamic surgery was performed with AO-labeled tumor cells (AO-PDS) by using an ultrasonic surgical knife (Olympus, Tokyo, Japan) and a fluorescence surgical microscope (Carl Zeiss, Oberkochen, Germany). Tumor fluorovisualization was performed after local administration of $1 \mu \mathrm{g} / \mathrm{ml}$ of AO solution (A8097: Sigma Aldrich Co., St. Louis, MO) for $5 \mathrm{~min}$, followed by washing out the excess $\mathrm{AO}$ solution with saline and excitation with blue light, which most effectively excites AO to emit green fluorescence [25]. Microscopic curettage was repeated until the green fluorescence (tumor cells) had disappeared. After AO-PDS, AO-PDT was performed [13-18]. AO-PDT was applied to the area of tumor curettage under illumination with $>100,000 \mathrm{~lx}$ of unfiltered light (visible light) from a xenon lamp for $10 \mathrm{~min}$, again using fluorescence surgical microscopy. After the curettage, bone reconstruction was performed by using magnetic materials $\left(\mathrm{Fe}_{3} \mathrm{O}_{4}\right.$ powder with calcium phosphate cement) for EMHT [23,24]. Finally, radiodynamic treatment, in which labeled cells were treated with low dose $\mathrm{X}$-rays (AO-RDT) was performed after closure of the surgical wound without washing out the AO solution. In the radiotherapy room, a single session of X-ray irradiation was applied to the resected area to achieve a strong cytocidal effect of AO excited by low-dose X-rays [22]. Tumor cells labeled with AO can be killed by low-dose radiation.

\subsection{Hyperthermia Treatment with an Electronic Magnetic Generator (EMHT)}

The concept of EMHT is that the thermal heating of the magnetic material by the electronic generator kills the tumor cells and that the electronic stimulation by the magnetic field induces bone formation [23,24]. For the clinical application of EMHT, magnetic materials are used. Magnetic material hyperthermia was postoperatively applied by inserting the affected limb into a cylindrical coil of the electromagnetic field generator. EMHT was performed postoperatively on days $8,10,12,15,17$, $19,22,24,26$ and 29 for 30 minutes.

\subsection{Patient Distribution}

Six patients with bone metastasis (7 locations) in their extremities were treated with AO therapy and EMHT combination therapy. The patient distribution is shown in Table 1. The average patient age was 62 years old (range: 56 - 70 years). The cancer types were renal cancer $(n=2)$, bladder cancer $(n=1)$, lung cancer $(n=1)$, and multiple myeloma $(n=1)$. The location of bone metastasis was distal humerus $(\mathrm{n}=4)$, distal femur $(\mathrm{n}=1)$, proximal tibia $(\mathrm{n}=1)$, and distal tibia $(\mathrm{n}=1)$.

\section{Results}

Curettage to remove the tumor was performed in all cases. Six patients received bone reconstruction with $\mathrm{Fe}_{3} \mathrm{O}_{4}$ powder and calcium phosphate or bone cement, and 1 patient with a distal femur metastasis received reconstruction with a distal femur tumor prosthesis (Table 1).

All study patients died during the study period. None of the deaths were related to the combination procedure. The mean postoperative survival time was 13 months (range, 6 - 33 months). There were no deaths during the immediate postoperative period. All patients received 
A Minimally Invasive Surgery for Bone Metastases Using the Combination of

Photodynamic Therapy and Hyperthermia Treatment

Table 1. Patient distribution, surgical procedures and clinical outcome.

\begin{tabular}{|c|c|c|c|c|c|c|c|c|c|}
\hline No. & $\begin{array}{c}\text { Age } \\
\text { (years) }\end{array}$ & Diagnosis & Metastasis site & Pattern of excision & $\begin{array}{l}\text { Reconstruction } \\
\text { method }\end{array}$ & $\begin{array}{l}\text { Follow } \\
\text { up }\end{array}$ & $\begin{array}{l}\text { Local } \\
\text { recurrence }\end{array}$ & Outcome & $\begin{array}{l}\text { Bony } \\
\text { union }\end{array}$ \\
\hline 1 & 56 & $\begin{array}{l}\text { Bladder } \\
\text { cancer }\end{array}$ & Dis-Humerus & Curettage & $\mathrm{FeO}_{4}+\mathrm{Hap}^{*}$ & 8 & $(-)$ & DOD & + \\
\hline 2 & 63 & $\begin{array}{l}\text { Lung } \\
\text { cancer }\end{array}$ & Dis-Humerus & Curettage & $\mathrm{FeO}_{4}+\mathrm{Hap}^{*}$ & 21 & 13 months & DOD & + \\
\hline 3 & 68 & $\begin{array}{l}\text { Renal } \\
\text { cancer }\end{array}$ & Dis-Humerus & Curettage & $\mathrm{FeO}_{4}+\mathrm{Hap}^{*}$ & 6 & $(-)$ & DOD & moderate \\
\hline 4 & 70 & $\begin{array}{l}\text { Liver } \\
\text { cancer }\end{array}$ & Dis-Humerus & Curettage & $\mathrm{FeO}_{4}+\mathrm{Hap}^{*}$ & 28 & $(-)$ & DOD & + \\
\hline 5 & 57 & $\begin{array}{l}\text { Multiple } \\
\text { myeloma }\end{array}$ & Prox-Tibia & Curettage & $\begin{array}{l}\mathrm{FeO}_{4}+\mathrm{Hap}^{*} \\
+ \text { Nail }\end{array}$ & 33 & $(-)$ & DOD & + \\
\hline \multirow[t]{2}{*}{6} & 61 & $\begin{array}{l}\text { Renal } \\
\text { cancer }\end{array}$ & $\begin{array}{l}\text { Dis-Femur/ } \\
\text { Shaft of Femur }\end{array}$ & $\begin{array}{l}\text { Marginal resection/ } \\
\text { Curettage }\end{array}$ & $\begin{array}{l}\text { Tumor } \\
\text { prosthesis }\end{array}$ & 10 & $(-)$ & DOD & / \\
\hline & “ & “ & Dis-Tibia & Curettage & $\mathrm{FeO}_{4}+\mathrm{PMMA}$ & 8 & $(-)$ & “ & / \\
\hline
\end{tabular}

Dis-, distal; Prox-, proximal; $\mathrm{FeO}_{4}$, metal powder of $\mathrm{FeO}_{4} ;{ }^{*}$ Hap, Hydroxyapatite paste; PMMA, polymethyl methacrylate; DOD, death of disease.

combination therapy with an air tourniquet and then showed minimal operative blood loss (mean, $80 \mathrm{ml}$; (range, $40-300 \mathrm{ml}$ ). In the follow-up period, only one patient experienced a local recurrence, and this recurrence occurred 13 months after surgery. EMHT was repeatedly performed for the recurrence case, and tumor reprogression was suppressed until the end of the patient's life.

Bony union occurred in 4 of 5 patients $(80 \%)$, and the limb function of all patients 2 months after surgery was assessed as excellent or good by the Enneking limb functional score.

The relief of pain was successful in all patients at an early postoperative period. At two weeks after surgery, all patients reported minimal or no pain. The range of motion (ROM) in adjacent joints showed no limitations, except for one case in which reconstruction was performed with a distal femur prosthesis.

There were no surgical complications. There were no neurological injuries, vascular injuries, or infections from this combination therapy.

\subsection{Case Presentation 1}

Case 1 was a 57 -year-old man who had multiple myeloma at the proximal tibia (Figure 1(A)). The patient had severer lower leg pain and could no longer walk. The osteolytic lesion extended outside the cortex, and there was a risk of pathological fracture. The osteolytic lesion at the tibia was treated with curettage following AOT with an ultrasonic knife. After curettage, reconstruction with $\mathrm{Fe}_{3} \mathrm{O}_{4}$ powder, calcium phosphate cement, and an intramedullary nail was performed (Figure 1(B)). Two months after undergoing EMHT, new bone formation was visi-

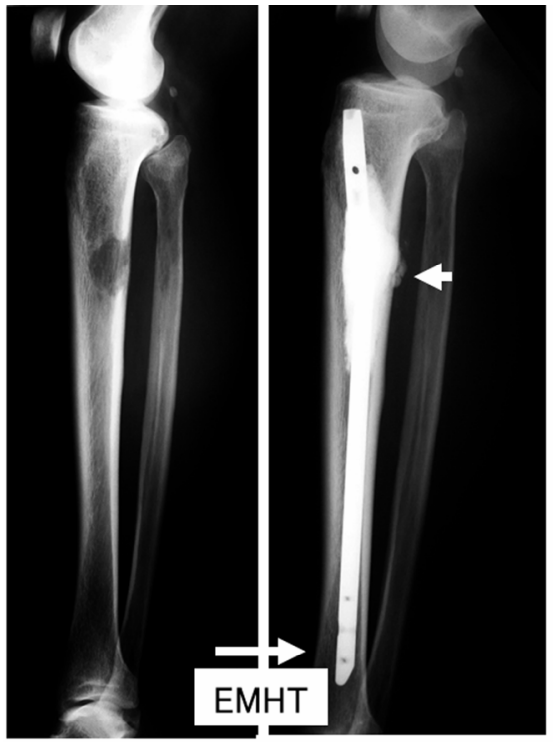

A

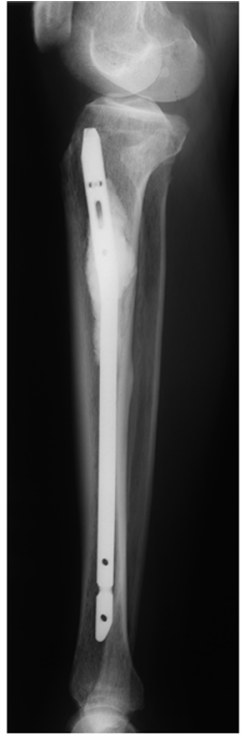

C
Figure 1. X-ray images of a multiple myeloma metastasis at the proximal tibia that destroyed part of the cortex (A). Two months after surgery, bone formation was identified (B; white arrow). No osteolytic change was identified (C) for 34 months. Limb function was preserved, and the patient was able to walk without pain until the time of death.

ble, and the patient walked smoothly without any pain. Local tumor regrowth was not seen during the follow-up period. The patient died of multiple myeloma 33 months after surgery (Figure 1(C)).

\subsection{Case Presentation 2}

Case 2 was a 68-year-old man who had distal humerus bone metastasis with renal cancer (Figure 2(A)). The 
bone metastasis extended from the distal humerus into the bone marrow around the middle of the humerus. $\mathrm{Cu}$ rettage following AOT with an ultrasonic knife was performed through the window of the distal cortex. Tumor labeled with $\mathrm{AO}$ can be visible with green fluorescence (Figures 3(A) and (B)). After curettage, reconstruction with the $\mathrm{Fe}_{3} \mathrm{O}_{4}$ powder with calcium phosphate cement was performed (Figure 2(B)). Three months after undergoing EMHT, new bone formation had become visible, and limb function was preserved, although the other metastasis progressed, and the patient died 6 months after surgery (Figure 2(C)).

\section{Discussion}

Bone metastases occur in the advanced stages of cancers such as lung, renal, and breast cancers. Pathological fractures caused by bone metastasis are associated with limited life expectancy, and the pathological fracture itself is a negative risk factor for prognosis [26]. Multiple metastases indicate poor prognosis. The goals of surgical intervention for bone metastases are pain relief and mobil-

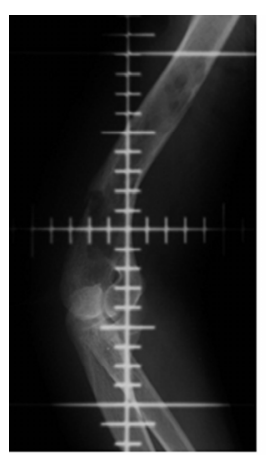

A

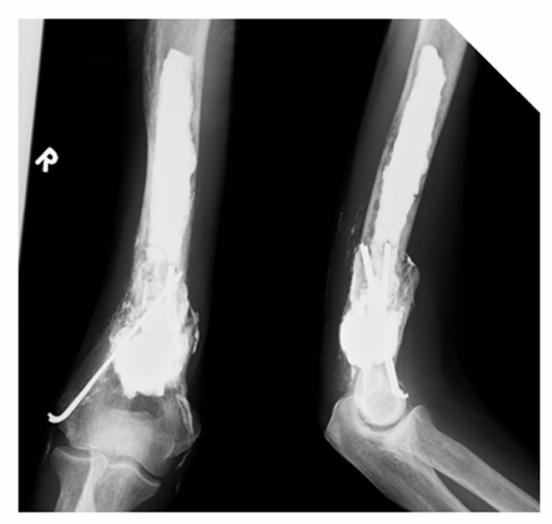

C

B

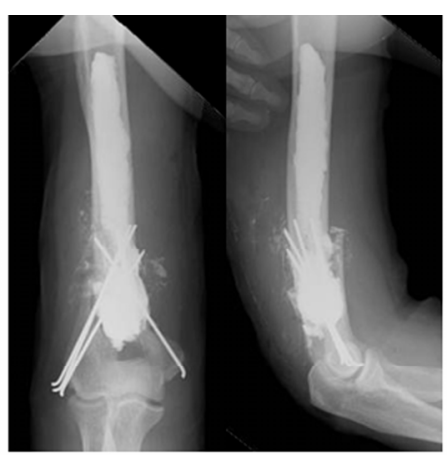

Figure 2. X-ray image for AO-RDT before surgery (A). The
tumor extended into the middle of the humerus. The area was curettaged and packed with hydroxyapatite paste and $\mathrm{FeO}_{4}$ powder (B). X-ray image 6 months after surgery. A callus appeared on the $\mathrm{X}$-ray, and there was no local recurrence for the remainder of the patient's life (C).

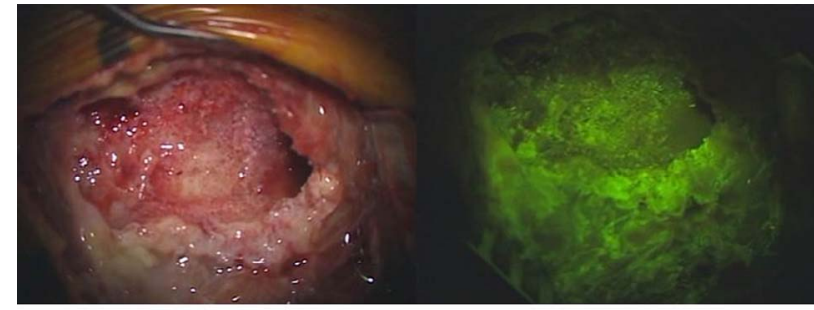

A

B

Figure 3. The microscopic views in the surgical fields through the optical light (A) and fluorescence with Acridine Orange (B). Tumor labeled with AO can be visible with green fluorescence.

ity preservation.

Therapeutic treatments for bone metastases to extremities include intramedullary nails, plates combined with bone cement and tumor prosthesis [1-7]. Rigid intramedullary nailing for bone metastasis is a convenient and effective method of stabilization [4]. In cases of bone metastasis at the diaphysis, we achieved good local control of bone metastasis by using intramedullary fixation before EMHT [23,24].

On the other hand, bone metastasis at the epiphysis sometimes requires surgical intervention without an intramedullary nail. The cortex at the epiphysis is thinner than at the diaphysis, and it is difficult to use an intramedullary nail. Around the epiphysis lesion, tumor curettage or resection is performed, and plates or pros-theses are used. However, the use of a tumor prosthesis is costly and more invasive than curettage with plates or bone cement, which is commonly performed [27]. Local adjuvants most commonly used for improving the effect of curettage in local cancer surgery may exert their effects either chemically or physically. In orthopaedic oncology, the most commonly used adjuvants are phenol, liquid nitrogen, and laser [27]. Liquid nitrogen sometimes causes bone fracture or skin erosion; the effect of laser treatment is limited to the bone surface; and phenol has toxicity. Epiphysial bone metastasis is an adequate indication for AO therapy, because the effect of AO therapy reaches deep areas and has fewer surgical complications.

We reported that $\mathrm{AO}$ accumulates in malignant tumors with low extracellular $\mathrm{pH}$ [28]. Cancer bone metastases in clinical specimens have a low extracellular $\mathrm{pH}$ (5.9 6.8) and high fluorescence intensity for AO. Thus, cancer bone metastasis is an adequate application for AO therapy.

AO therapy includes AO radiodynamic therapy with low-dose (5 Gy) radiation. Regardless of whether the course of treatment for metastatic bone lesions is nonoperative or operative, conventional radiation therapy is mandatory for local tumor control and effective pain re- 
lief. Disease progression occurs in approximately $15 \%$ to $20 \%$ of patients with only surgery at the humerus [29]. Evidence indicating the best radiation dose fraction schedule is lacking. Higher doses may promote local control, but also increase complications due to radiation toxicity. AO-RDT uses the dose of only 5 Gy, because AO increases radiation sensitivity of the tumor cells. AO-RDT accelerates the effect of radiation therapy without the increased complications of conventional high-dose radiation therapy.

Radiation therapy for bone metastasis without surgical intervention is a risk for pathological fracture [7,8]. Furthermore, radiation therapy cannot repeatedly be performed, due to the development of tolerance.

EMHT has the advantage of reducing complications of radiotherapy. Radiation therapy inhibits bone formation, due to the death of osteoblasts. On the other hand, hyperthermia by EMHT kills the tumor cells, EMHT can repeatedly be performed without increased complications (unlike conventional radiation therapy), and EMHT has the potential to promote bone formation by electronic stimulation [30-33]. We previously reported the efficacy of hyperthermia treatment for bone metastasis compared with the surgical treatment in combination with or without radiotherapy [23]. The 8 patients who underwent only palliative operation without radiotherapy showed poor local control, and the radiographic assessment showed the significant difference between palliative operation alone and the EMHT alone. The palliative operation group showed $38 \%$ bony union, in contrast with $80 \%$ bony union in this study [23]. Thus, EMHT can reduce the complications of radiation therapy and has the potential to promote bone union for the cancer bone metastasis.

Furthermore, hyperthermia promotes the chemotherapy effect of thermal heating [34-36]. EMHT can be performed without interrupting post-surgical chemotherapy and has the potential to promote the effect of chemotherapy on bone metastasis lesions.

Clinical results of our combined therapy showed that only one patient experienced local recurrence; this recurrence occurred 13 months after surgery. Thus, the local recurrence was controlled by EMHT. Complete bony union occurred in $80 \%$ of cases, and excellent local control was achieved even though all cases had a positive surgical margin. Because the surrounding normal tissue was preserved, the limb function after surgery was assessed as excellent in 5 of 6 cases.

For patients with multiple bone metastases, a reduction surgical procedure is generally performed, because cancer is a systemic disease, and local treatment of bone metastasis can preserve the patients' quality of life.

Cancers often become tolerant to chemotherapy or radiation therapy, and this tolerance allows the disease stage to progress. In many cases of local recurrence of bone metastases, additional surgery cannot be performed due to the patient's deteriorating condition caused by advanced disease. For these patients, EMHT would serve as a repeatable and noninvasive treatment suitable for bone metastasis.

There are several additional advantages of AO therapy combined with EMHT: 1) Tumors can be visualized by fluorescent microscopy during surgery; 2) Visible cells can be removed without damage to healthy tissues; and 3) Residual AO-labelled tumor cells seated in deep areas are killed by low-dose radiation after surgery. Because of these advantages, the combination of AO therapy and EMHT has the potential to replace conventional tumor surgery and radiotherapy in some cases.

In conclusion, minimally invasive treatment of patients with bone metastasis with AO therapy and EMHT combination therapy is an effective and safe procedure. This surgical method is associated with a low rate of complications. Our goal of preserving excellent limb function with low risk of local tumor recurrence in bone metastasis was mostly achieved. However, we studied only a small number of patients. A future study with a large number of patients is needed to confirm the clinical utility of AO therapy combined with EMHT in cancer treatment.

\section{Acknowledgements}

We thank Dr. Haruhiko Satonaka (Ise Municipal General Hospital, Ise City, Mie, Japan) for technical and basic research assistance.

\section{REFERENCES}

[1] P. Bohm and J. Huber, "The Surgical Treatment of Bony Metastases of the Spine and Limbs," The Journal of Bone and Joint Surgery. British Volume, Vol. 84, No. 4, 2002, pp. 521-529. doi:10.1302/0301-620X.84B4.12495

[2] R. E. Coleman, "Clinical Features of Metastatic Bone Disease and Risk of Skeletal Morbidity," Clinical Cancer Research, Vol. 12, No. 20, 2006, pp. 6243s-6249s. doi:10.1158/1078-0432.CCR-06-0931

[3] D. J. Jacofsky, P. J. Papagelopoulos and F. H. Sim, “Advances and Challenges in the Surgical Treatment of Metastatic Bone Disease," Clinical Orthopaedics and Related Research, No. 415, 2003, pp. S14-S18.

[4] O. Ofluoglu, B. Erol, Z. Ozgen and M. Yildiz, "Minimally Invasive Treatment of Pathological Fractures of the Humeral Shaft," International Orthopaedics, Vol. 33, No. 3, 2009, pp. 707-712. doi:10.1007/s00264-008-0540-0

[5] C. M. Ogilvie, E. J. Fox and R. D. Lackman, "Current Surgical Management of Bone Metastases in the Extremities and Pelvis," Seminars in Oncology, Vol. 35, No. 2, 2008, pp. 118-128. doi:10.1053/j.seminoncol.2007.11.019 
[6] S. Utzschneider, E. Wicherek, P. Weber, G. Schmidt, V. Jansson and H. R. Durr, "Surgical Treatment of Bone Metastases in Patients with Lung Cancer," International Orthopaedics, Vol. 35, No. 5, 2011, pp. 731-736. doi:10.1007/s00264-010-1074-9

[7] J. S. Keene, D. S. Sellinger, A. A. McBeath and W. D. Engber, "Metastatic Breast Cancer in the Femur. A Search for the Lesion at Risk of Fracture," Clinical Orthopaedics and Related Research, No. 203, 1986, pp. 282-288.

[8] Y. M. Van der Linden, P. D. Dijkstra, H. M. Kroon, J. J. Lok, E. M. Noordijk, J. W. Leer and C. A. Marijnen, "Comparative Analysis of Risk Factors for Pathological Fracture with Femoral Metastases," The Journal of Bone and Joint Surgery. British Volume, Vol. 86, No. 4, 2004, pp. 566-573.

[9] K. Kusuzaki, S. Hosogi, E. Ashihara, T. Matsubara, H. Satonaka, T. Nakamura, A. Matsumine, A. Sudo, A. Uchida, H. Murata, N. Baldini, S. Fais and Y. Marunaka, "Translational Research of Photodynamic Therapy with Acridine Orange Which Targets Cancer Acidity," Current Pharmaceutical Design, Vol. 18, No. 10, 2012, pp. 14141420. doi:10.2174/138161212799504812

[10] K. Kusuzaki, H. Murata, T. Matsubara, S. Miyazaki, A. Okamura, M. Seto, A. Matsumine, H. Hosoi, T. Sugimoto and A. Uchida, "Clinical Trial of Photodynamic Therapy Using Acridine Orange with/without Low Dose Radiation as New Limb Salvage Modality in Musculoskeletal Sarcomas," Anticancer Research, Vol. 25, No. 2B, 2005, pp. 1225-1235.

[11] K. Kusuzaki, H. Murata, T. Matsubara, S. Miyazaki, K. Shintani, M. Seto, A. Matsumine, H. Hosoi, T. Sugimoto and A. Uchida, "Clinical Outcome of a Novel Photodynamic Therapy Technique Using Acridine Orange for Synovial Sarcomas," Photochemistry and Photobiology, Vol. 81, No. 3, 2005, pp. 705-709. doi:10.1562/2004-06-27-RA-218

[12] K. Kusuzaki, H. Murata, T. Matsubara, H. Satonaka, T. Wakabayashi, A. Matsumine and A. Uchida, "Review. Acridine Orange Could Be an Innovative Anticancer Agent under Photon Energy," In Vivo (Athens, Greece), Vol. 21, No. 2, 2007, pp. 205-214.

[13] T. Matsubara, K. Kusuzaki, A. Matsumine, H. Murata, T. Nakamura, A. Uchida and A. Sudo, "Clinical Outcomes of Minimally Invasive Surgery Using Acridine Orange for Musculoskeletal Sarcomas around the Forearm, Compared with Conventional Limb Salvage Surgery after Wide Resection," Journal of Surgical Oncology, Vol. 102, No. 3, 2010, pp. 271-275. doi:10.1002/jso.21602

[14] T. Matsubara, K. Kusuzaki, A. Matsumine, H. Murata, H. Satonaka, K. Shintani, T. Nakamura, H. Hosoi, T. Iehara, T. Sugimoto and A. Uchida, "A New Therapeutic Modality Involving Acridine Orange Excitation by Photon Energy Used during Reduction Surgery for Rhabdomyosarcomas," Oncology Reports, Vol. 21, No. 1, 2009, pp. 8994.

[15] T. Matsubara, K. Kusuzaki, A. Matsumine, T. Nakamura and A. Sudo, "Can a Less Radical Surgery Using Photo- dynamic Therapy With Acridine Orange Be Equal to a Wide-Margin Resection?" Clinical Orthopaedics and Related Research, Vol. 471, No. 3, 2013, pp. 792-802. doi:10.1007/s11999-012-2616-9

[16] T. Nakamura, K. Kusuzaki, T. Matsubara, A. Matsumine, H. Murata and A. Uchida, "A New Limb Salvage Surgery in Cases of High-Grade Soft Tissue Sarcoma Using Photodynamic Surgery, Followed by Photo- and Radiodynamic Therapy with Acridine Orange," Journal of Surgical Oncology, Vol. 97, No. 6, 2008, pp. 523-528. doi:10.1002/jso.21025

[17] K. Yoshida, K. Kusuzaki, T. Matsubara, A. Matsumine, T. Kumamoto, Y. Komada, N. Naka and A. Uchida, "Periosteal Ewing's Sarcoma Treated by Photodynamic Therapy with Acridine Orange," Oncology Reports, Vol. 13, No. 2, 2005, pp. 279-282.

[18] K. Kusuzaki, K. Aomori, T. Suginoshita, G. Minami, H. Takeshita, H. Murata, S. Hashiguchi, T. Ashihara and Y. Hirasawa, "Total Tumor Cell Elimination with Minimum Damage to Normal Tissues in Musculoskeletal Sarcomas Following Photodynamic Therapy with Acridine Orange," Oncology, Vol. 59, No. 2, 2000, pp. 174-180.

[19] K. Kusuzaki, G. Minami, H. Takeshita, H. Murata, S. Hashiguchi, T. Nozaki, T. Ashihara and Y. Hirasawa, "Photodynamic Inactivation with Acridine Orange on a Multidrug-Resistant Mouse Osteosarcoma Cell Line," Japanese Journal of Cancer Research, Vol. 91, No. 4, 2000, pp. 439-445. doi:10.1111/j.1349-7006.2000.tb00964.x

[20] K. Kusuzaki, H. Murata, H. Takeshita, S. Hashiguchi, T. Nozaki, K. Emoto, T. Ashihara and Y. Hirasawa, "Intracellular Binding Sites of Acridine Orange in Living Osteosarcoma Cells," Anticancer Research, Vol. 20, No. 2A, 2000, pp. 971-975.

[21] K. Kusuzaki, T. Suginoshita, G. Minami, K. Aomori, H. Takeshita, H. Murata, S. Hashiguchi, T. Ashihara and Y. Hirasawa, "Fluorovisualization Effect of Acridine Orange on Mouse Osteosarcoma," Anticancer Research, Vol. 20, No. 5A, 2000, pp. 3019-3024.

[22] S. Hashiguchi, K. Kusuzaki, H. Murata, H. Takeshita, M. Hashiba, T. Nishimura, T. Ashihara and Y. Hirasawa, "Acridine Orange Excited by Low-Dose Radiation Has a Strong Cytocidal Effect on Mouse Osteosarcoma," Oncology, Vol. 62, No. 1, 2002, pp. 85-93. doi:10.1159/000048251

[23] A. Matsumine, K. Kusuzaki, T. Matsubara, K. Shintani, H. Satonaka, T. Wakabayashi, S. Miyazaki, K. Morita, K. Takegami and A. Uchida, "Novel Hyperthermia for Metastatic Bone Tumors with Magnetic Materials by Generating an Alternating Electromagnetic Field," Clinical \& Experimental Metastasis, Vol. 24, No. 3, 2007, pp. 191200. doi:10.1007/s10585-007-9068-8

[24] A. Matsumine, K. Takegami, K. Asanuma, T. Matsubara, T. Nakamura, A. Uchida and A. Sudo, "A Novel Hyperthermia Treatment for Bone Metastases Using Magnetic Materials," International Journal of Clinical Oncology, Vol. 16, No. 2, 2011, pp. 101-108. doi:10.1007/s10147-011-0217-3

[25] H. Ueda, H. Murata, H. Takeshita, G. Minami, S. Hashi- 
guchi and T. Kubo, "Unfiltered Xenon Light Is Useful for Photodynamic Therapy with Acridine Orange," Anticancer Research, Vol. 25, No. 6B, 2005, pp. 3979-3983.

[26] H. Katagiri, M. Takahashi, K. Wakai, H. Sugiura, T. Kataoka and K. Nakanishi, "Prognostic Factors and a Scoring System for Patients with Skeletal Metastasis," The Journal of Bone and Joint Surgery. British Volume, Vol. 87, No. 5, 2005, pp. 698-703. doi:10.1302/0301-620X.87B5.15185

[27] A. Piccioli, A. Ventura, G. Maccauro, M. S. Spinelli, V. Del Bravo and M. A. Rosa, "Local Adjuvants in Surgical Management of Bone Metastases," International Journal of Immunopathology and Pharmacology, Vol. 24, No. 1, 2011, pp. 129-132.

[28] T. Matsubara, K. Kusuzaki, A. Matsumine, K. Shintani, H. Satonaka and A. Uchida, "Acridine Orange Used for Photodynamic Therapy Accumulates in Malignant Musculoskeletal Tumors Depending on $\mathrm{pH}$ Gradient," Anticancer Research, Vol. 26, No. 1A, 2006, pp. 187-193.

[29] F. J. Frassica and D. A. Frassica, "Evaluation and Treatment of Metastases to the Humerus," Clinical Orthopaedics and Related Research, No. 415, 2003, pp. S212-S218. doi:10.1097/01.blo.0000093052.96273.a7

[30] S. Adie, I. A. Harris, J. M. Naylor, H. Rae, A. Dao, S. Yong and V. Ying, "Pulsed Electromagnetic Field Stimulation for Acute Tibial Shaft Fractures: A Multicenter, Double-Blind, Randomized Trial," The Journal of Bone and Joint Surgery, American Volume, Vol. 93, No. 17, 2011, pp. 1569-1576. doi:10.2106/JBJS.J.00869

[31] X. L. Griffin, M. L. Costa, N. Parsons and N. Smith, "Electromagnetic Field Stimulation for Treating Delayed Union or Non-Union of Long Bone Fractures in Adults," Cochrane Database of Systematic Reviews (Online), No. 4, 2011, Article ID: CD008471. doi:10.1002/14651858.CD008471.pub2

[32] P. Hannemann, K. W. Gottgens, B. J. van Wely, K. A. Kolkman, A. J. Werre, M. Poeze and P. R. Brink, "Pulsed
Electromagnetic Fields in the Treatment of Fresh Scaphoid Fractures. A Multicenter, Prospective, Double Blind, Placebo Controlled, Randomized Trial," BMC Musculoskeletal Disorders, Vol. 12, 2011, p. 90. doi:10.1186/1471-2474-12-90

[33] H. F. Shi, J. Xiong, Y. X. Chen, J. F. Wang, X. S. Qiu, Y. H. Wang and Y. Qiu, "Early Application of Pulsed Electromagnetic Field in the Treatment of Postoperative Delayed Union of Long-Bone Fractures: A Prospective Randomized Controlled Study," BMC Musculoskeletal Disorders, Vol. 14, 2013, p. 35. doi:10.1186/1471-2474-14-35

[34] S. Adachi, S. Kokura, T. Okayama, T. Ishikawa, T. Takagi, O. Handa, Y. Naito and T. Yoshikawa, "Effect of Hyperthermia Combined with Gemcitabine on Apoptotic Cell Death in Cultured Human Pancreatic Cancer Cell Lines," International Journal of Hyperthermia, Vol. 25, No. 3, 2009, pp. 210-219. doi:10.1080/02656730802657036

[35] T. Ishikawa, S. Kokura, N. Sakamoto, T. Ando, E. Imamoto, T. Hattori, H. Oyamada, N. Yoshinami, M. Sakamoto, K. Kitagawa, Y. Okumura, N. Yoshida, K. Kamada, K. Katada, K. Uchiyama, O. Handa, T. Takagi, H. Yasuda, J. Sakagami, H. Konishi, N. Yagi, Y. Naito and T. Yoshikawa, "Phase II Trial of Combined Regional Hyperthermia and Gemcitabine for Locally Advanced or Metastatic Pancreatic Cancer," International Journal of Hyperthermia, Vol. 28, No. 7, 2012, pp. 597-604. doi:10.3109/02656736.2012.695428

[36] T. Okayama, S. Kokura, T. Ishikawa, S. Adachi, T. Hattori, T. Takagi, O. Handa, Y. Naito and T. Yoshikawa, "Antitumor Effect of Pretreatment for Colon Cancer Cells with Hyperthermia Plus Geranylgeranylacetone in Experimental Metastasis Models and a Subcutaneous Tumor Model of Colon Cancer in Mice," International Journal of Hyperthermia, Vol. 25, No. 2, 2009, pp. 141-149. doi: $10.1080 / 02656730802631783$ 